\title{
Estudo químico e da atividade biológica cardiovascular do óleo essencial de folhas de Alpinia zerumbet (Pers.) B.L.Burtt \& R.M.Sm. em ratos
}

\author{
BARCELOS, F.F.; OLIVEIRA, M.L.; GIOVANINNI, N.P.B.; LINS, T.P.; FILOMENO, C.A.; SCHNEIDER, S.Z.; \\ PINTO, V.D.; ENDRINGER, D.C.; ANDRADE, T.U. \\ Departamento de Farmácia, Centro Universitário Vila Velha - UVV, CEP: 29102-770, Vila Velha-Brasil \\ *tadeu.andrade@uvv.br
}

\begin{abstract}
RESUMO: A espécie vegetal Alpinia zerumbet (Pers.) B.L.Burtt \& R.M. Sm. é popularmente empregada para o tratamento de diversas enfermidades, entre elas a hipertensão. Avaliar a composição química, a atividade antihipertensiva e ação na hipertrofia cardíaca do óleo essencial das folhas de Alpinia zerumbet (OEAZ) em ratos foram os objetivos deste estudo. O OEAZ, obtido por hidrodestilação em aparelho Clevenger, teve sua composição química analisada em cromatografia gasosa acoplada à espectrometria de massas (CG-EM). Foram identificados 14 constituintes, sendo terpinen-4-ol (37,45\%) o majoritário, seguido pelos óxido de cariofileno (7,56\%), trans-hidrato de sabineno (6,61\%) e 1,8-cineol (4,02\%). A avaliação cardiovascular foi feita após o tratamento crônico de ratos espontaneamente hipertensos (SHR) e seus respectivos controles, ratos Wistar-Kyoto (WKY). Os dados hemodinâmicos revelaram redução da pressão arterial média (PAM) no grupo tratado (SHRP: $160 \pm 7 \mathrm{~mm} \mathrm{Hg} ; \mathrm{p}<0,01$ ) em relação ao não tratado (SHR: $180 \pm 5 \mathrm{~mm} \mathrm{Hg}$ ). A relação entre peso do ventrículo esquerdo e peso corporal (VE/PC) do SHRP $\left(2,50 \pm 0,03 \mathrm{mg} \mathrm{g}^{-1} ; p<0,01\right)$ mostrou-se inferior ao SHR $\left(2,61 \pm 0,01 \mathrm{mg} \mathrm{g}^{-1}\right)$, confirmando a redução da hipertrofia cardíaca $(\mathrm{HC})$. Os dados de PAM e VE/PC dos animais SHRP foram estatisticamente diferentes quando comparados com os ratos controle (WKY: $116 \pm 2 \mathrm{~mm} \mathrm{Hg} \mathrm{e}$ WKYP: $119 \pm 4 \mathrm{~mm} \mathrm{Hg}$; $p<0,05 ;$ WKY: 2,15 $\pm 0,04 \mathrm{mg} \mathrm{g}^{-1}$ e WKYP: 2,17 $\left.\pm 0,04 \mathrm{mg} \mathrm{g}^{-1} ; p<0,01\right)$, indicando não ter havido normalização dos mesmos. Conclui-se que o tratamento crônico com OEAZ foi capaz de determinar redução, mas não a normalização, da PAM e da HC de ratos SHR, provavelmente pela presença dos componentes terpinen-4-ol e 1,8-cineol. Estudos com doses maiores ou período de tratamento superior são necessários para avaliar a possibilidade de 0 OEAZ normalizar os parâmetros analisados (PAM e HC).
\end{abstract}

Palavras-chave: óleo essencial, terpinen-4-ol, 1,8-cineol, Alpinia zerumbet, hipertensão, SHR

\begin{abstract}
Phytochemistry and cardiovascular biological activity of the essential oil from leaves of Alpinia zerumbet (Pers.) B.L. Burtt \& R.M.Sm. in rats. Alpinia zerumbet (Pers.) B.L. Burtt \& R.M.Sm. is traditionally employed to treat several diseases such as hypertension. The aim of this study was to evaluate the chemical composition, the anti-hypertensive activity and the capacity to reduce cardiac hypertrophy of the essential oil of $A$. zerumbet leaves (EOAZ) in rats. EOAZ was obtained through hydrodistillation in Clevenger apparatus and its chemical composition was analyzed by gas chromatography-mass spectrometry (GC-MS). Several constituents (14) were identified, terpen-4-ol (37.45\%) being the major component, followed by caryophyllene oxide $(7.56 \%)$, trans-sabinene hydrate $(6.61 \%)$ and 1,8 -cineol $(4.02 \%)$. The cardiovascular effect was investigated after chronic treatment with spontaneously hypertensive rats (SHR) and their respective controls, Wistar-Kyoto rats (WKY). The treated group showed a lower mean arterial pressure (MAP) (SHRP: $160 \pm 7 \mathrm{~mm} \mathrm{Hg} ; \mathrm{p}<0.01$ ) than the untreated group (SHR: $180 \pm 5 \mathrm{~mm} \mathrm{Hg}$ ). The ratio of left ventricle-to-body weight (LV/BW) for SHRP was lower $\left(2.504 \pm 0.03 \mathrm{mg} \mathrm{g}^{-1} ; \mathrm{p}<0.01\right)$ than that for SHR $\left(2.162 \pm 0.01 \mathrm{mg} \mathrm{g}^{-1}\right)$, confirming the cardiac hypertrophy $(\mathrm{CH})$ reduction. There were significant differences in MAP and $\mathrm{CH}$ between SHRP animals and control rats (WKY: $116 \pm 2 \mathrm{~mm} \mathrm{Hg}$ and WKYP: $119 \pm 4 \mathrm{~mm} \mathrm{Hg} ; \mathrm{p}<0.05$. WKY: 2.152
\end{abstract}

Recebido para publicação em 02/12/2008

Aceito para publicação em 11/07/2009

Rev. Bras. PI. Med., Botucatu, v.12, n.1, p.48-56, 2010. 
$\pm 0.04 \mathrm{mg} \mathrm{g}^{-1}$ and WKYP: $\left.2.168 \pm 0.04 \mathrm{mg} \mathrm{g}^{-1} ; \mathrm{p}<0.01\right)$, indicating that these values were not normalized. Those data showed that the chronic treatment with EOAZ reduces MAP and $\mathrm{CH}$ in SHR probably due to the presence of the compounds terpinen-4-ol and 1,8-cineol. Studies with higher doses or longer treatment periods are necessary to evaluate whether EOAZ can reduce the analyzed parameters (MAP and $\mathrm{CH}$ ) to normal values.

Key words: essential oil, terpinen-4-ol, 1,8-cineol, Alpinia zerumbet, hypertension, SHR

\section{INTRODUÇÃO}

O uso de plantas como recurso terapêutico pode ser traçado desde a antiguidade até os dias atuais (WHO, 2002; Lee, 2004). Estima-se que cerca de $80 \%$ da população dos países em desenvolvimento depende do uso de plantas medicinais para assistência primária à saúde, sendo vários os benefícios terapêuticos atribuídos a essas espécies vegetais (Hostettmann \& Marston 2002; Maciel et al., 2002; WHO, 2002). Esses fatos instigaram a pesquisa em diversas áreas, que envolvem as plantas medicinais, como agronomia, botânica, farmacologia e fitoquímica, visando fornecer dados que contribuíssem com o desenvolvimento e aperfeiçoamento da fitoterapia (Maciel et al., 2002), tornando-a recurso terapêutico alternativo viável para o tratamento para diversas enfermidades (WHO, 2002).

No Brasil, estima-se que o uso de espécies vegetais com fins medicinais é prática difundida, enriquecida pela diferenças culturais, provenientes dos índios, negros e europeus (Brandão, 1996). A sabedoria popular sobre plantas medicinais não deve ser subestimada, mas, no entanto, somente deve ser repassada como verdadeira para a população depois de confirmar se as atividades atribuídas às espécies vegetais realmente existem, e que o seu uso como medicamento é seguro (Brasil, 2007). Haja vista ser ainda insuficiente o número de estudos científicos acerca do assunto, torna-se necessário estimular a realização desses estudos, tendo em vista a importância dos seus resultados tanto individuais como sociais (Brasil, 2007).

A espécie Alpinia zerumbet (Pers.) B.L.Burtt \& R.M.Sm., nativa de regiões tropicais do sul e sudeste asiático, tendo a introdução no Brasil, no século XIX, ocorrida ou por acaso, por meio da mistura dos rizomas com a areia que servia de lastro às caravelas portuguesas que voltavam das Índias (Albuquerque \& Neves, 2004), ou proposital, quando a espécie fora trazida para o Jardim Botânico do Rio de Janeiro, para presentear a princesa Isabel, logo após a mesma ter assinado a Lei Áurea, em 13 de maio de 1888 (Pio Corrêa, 1984). Diversos são os nomes populares atribuídos à $A$. zerumbet, tais como pacová, colônia, paco-seroca, cuité-açu, gengibreconcha, dentre outros (Albuquerque \& Neves, 2004). Esta espécie pertence à família Zingiberaceae, sendo o gênero Alpinia o maior dessa família, com aproximadamente 230 espécies (Kress et al., 2005). $A$. zerumbeté erva terrestre com 1,5 a 2,5 metros de altura, apresentando folhas alternas, completas e simples, com caules aéreos e curtos agrupados em touceiras (Albuquerque \& Neves, 2004).

Popularmente, $A$. zerumbet é empregada como depurativa, diurética, hipotensora, antihistérica e vermífuga, sendo as folhas, flores e rizomas as partes vegetais mais utilizadas (Albuquerque et al., 2007; Oliveira \& Araújo, 2007). Essas potencialidades medicinais, que auxiliam na profilaxia e no tratamento de diversos males, vêm sendo estudadas por alguns grupos (Lahlou et al., 2002a; Lahlou et al., 2003; LealCardoso et al., 2004; Moura et al., 2005; Pinho et al., 2005).

A atividade hipotensora do óleo essencial de A. zerumbet (OEAZ) foi avaliada empregando-se diferentes ensaios para estudo dos mecanismos de ação (Lahlou et al., 2002a; Lahlou et al., 2003). OEAZ (1-20 $\left.\mathrm{mg} \mathrm{Kg}^{-1}\right)$ promoveu redução imediata e significante da pressão arterial de ratos normotensos (Lahlou et al., 2002a) e com hipertensão induzida por sal de deoxicorticosterona (DOCA-sal) (Lahlou et al., 2003). O principal constituinte de OEAZ, o terpinen4-ol, também foi avaliado no modelo de hipertensão DOCA-sal, apresentando diminuição da pressão arterial, dose-dependente (1-10 $\mathrm{mg} \mathrm{Kg}^{-1}$ ), indicando ser esse constituinte o responsável pela ação antihipertensiva do OEAZ (Lahlou et al., 2003). Nesse ensaio, os autores observaram que a atividade hipotensora do terpinen-4-ol, ocorreu independente da presença funcional do sistema nervoso simpático, sugerindo ação periférica, com vasodilatação (Lahlou et al., 2003).

Moura et al. (2005) demonstraram efeito antihipertensivo crônico de extrato hidroalcoólico de A. zerumbet, também utilizando o modelo de hipertensão DOCA-sal.

Para melhor compreender a fisiopatologia e a terapêutica da hipertensão arterial, uma linhagem de ratos espontaneamente hipertensos (SHR) fora selecionada, geneticamente, para o desenvolvimento de um modelo experimental (Okamoto \& Aoki, 1963). Ao nascer, os ratos SHR são normotensos, mas por volta da quinta semana de vida, a pressão sangüínea 
arterial eleva-se progressivamente, podendo atingir 220 $\mathrm{mm} \mathrm{Hg}$, mantendo-se assim durante toda a vida do animal, tornando-se o modelo experimental de maior utilidade para o estudo fisiopatológico da hipertensão essencial humana (National Research Council, 1976). Embora, estudos avaliando o efeito antihipertensivo crônico em ratos SHR sejam amplamente empregados, não é conhecido nenhum estudo que tenha sido conduzido com o óleo essencial de $A$. Zerumbet, empregando-se o referido modelo.

Desta forma, este estudo buscou investigar a existência de efeito hipotensor crônico do OEAZ e consequente redução da hipertrofia cardíaca em animais SHR, identificar os constituintes químicos do óleo essencial de folhas da $A$. zerumbet, bem como descrever a anatomia do material vegetal empregado.

\section{MATERIAL E MÉTODO}

\section{Material vegetal}

As folhas de $A$. zerumbet foram coletadas no mês de abril de 2008, no bairro da Prainha, em Vila Velha (ES), em horta parceira da Igreja Católica. A espécie foi identificada pela Profa M.Sc. Solange Z. Schneider, curadora do herbário do Centro Universitário Vila Velha - UVV, onde a exsicata encontra-se depositada, sob número UVVES 1982.

As folhas frescas da planta foram submetidas à análise histológica por microscopia, conforme métodos descritos por Bukatsh (1972) e Johansen (1940).

Inicialmente, realizou-se a triagem do material coletado, quando foram descartadas as folhas contaminadas por insetos ou fungos. O material não foi previamente lavado, sendo diretamente submetido à secagem em estufa ventilada, a $40^{\circ} \mathrm{C}$, por $72 \mathrm{~h}$.

\section{Obtenção do óleo essencial}

Empregou-se o método de hidrodestilação, em aparelho Clevenger (Logen, mod. LS 2015), ajustado por Skrubis (1982) e Ming et al. (1996), com aquecimento mantido na temperatura mínima necessária à ebulição. $\mathrm{O}$ aparelho de Clevenger foi adaptado a balão de fundo redondo com capacidade de 2,0 L, onde $1,5 \mathrm{~L}$ de água deionizada e a amostra vegetal $(20 \mathrm{~g})$ foram adicionadas. $O$ tempo de extração foi de duas horas, contadas a partir da ebulição da água. O hidrolato obtido foi particionado com pentano $(3 \times 50 \mathrm{~mL})$, a fração orgânica foi filtrada sobre sulfato de magnésio anidro. Esse filtrado foi submetido à evaporação do solvente em evaporador rotativo (Fisatom, mod. 802 ), a $30^{\circ} \mathrm{C}$ e pressão reduzida ( 40 $\mathrm{mm} \mathrm{Hg}$ ). Todo o sistema foi protegido da luz por meio de papel alumínio e o óleo essencial foi armazenado em frascos âmbar sob resfriamento. $O$ rendimento de extração foi determinado pela relação proporcional entre a massa das folhas e a massa do óleo obtido, sendo esse processo realizado em sextuplicata.

\section{Identificação e quantificação dos componentes químicos}

A identificação dos constituintes do óleo essencial (Adams,1995) foi realizada empregandose cromatografia gasosa, acoplada à espectrometria de massas (CG-EM. aparelho Shimadzu, modelo GCMS-QP 5050). Na identificação da constituição química foi empregada coluna DB-5HT (J \& W Scientific $($ )), com $30 \mathrm{~m}$ de comprimento, diâmetro interno de $0,32 \mathrm{~mm}$, espessura do filme de $0,10 \mu \mathrm{m}$, e nitrogênio como gás de arraste. As condições programadas no aparelho foram: pressão interna da coluna de $56,7 \mathrm{kPa}$, razão de split de 1:20, fluxo de gás na coluna de $1,0 \mathrm{~mL} \mathrm{~min}^{-1}\left(210^{\circ} \mathrm{C}\right)$, temperatura no injetor de $220^{\circ} \mathrm{C}$, temperatura no detector ou na interface (CG/EM) de $240^{\circ} \mathrm{C}$. A temperatura da [LC3] inicial da coluna foi $60^{\circ} \mathrm{C}$ por $1 \mathrm{~min}$, seguido de um incremento de $3^{\circ} \mathrm{C} \mathrm{min}^{-1}$ até atingir $240^{\circ} \mathrm{C}$, sendo mantida constante por 30 min. $O$ espectrômetro de massas foi programado para realizar leituras em uma faixa de 29 a $400 \mathrm{Da}$, em intervalos de $0,5 \mathrm{~s}$, com energia de ionização de $70 \mathrm{eV}$. Foram injetados $1 \mu \mathrm{L}$ de cada amostra, na concentração de 10.000 ppm, dissolvida em hexano. A identificação dos componentes foi feita pela comparação de seus espectros de massas com os disponíveis no banco de dados da espectroteca Willey 330.000 (7a ed.), e também pelos índices de Kovats. Para cálculo dos índices de Kovats, foi injetada no cromatógrafo a mistura de alcanos lineares (C9 a C26).

A quantificação dos componentes foi realizada utilizando-se cromatógrafo a gás com detector de ionização de chamas (GCFID) da marca Shimadzu, modelo GC-17A. As análises foram realizadas nas mesmas condições descritas para a identificação dos constituintes. Essas análises foram realizadas em triplicata.

\section{Grupos experimentais}

Foram utilizados ratos espontaneamente hipertensos (spontaneously hypertensive rats - SHR) e respectivos controles, os ratos Wistar-Kyoto (WKY), machos com três meses e com peso corporal variando entre $200-260 \mathrm{~g}$. Os animais foram mantidos em ambiente com iluminação artificial (ciclo claroescuro de $12 \mathrm{~h}$ ) e temperatura de $20-25^{\circ} \mathrm{C}$ de acordo com o recomendado pelos biotérios de pesquisa (FINEP). As gaiolas individuais permitiram o acesso dos animais à ingestão de água e ração (ração Probiotério, Moinho Primor, S.A) ad libitum. Como o volume de óleo essencial a ser aplicado era muito reduzido, optou-se pela utilização da via intraperitoneal.

Os animais foram divididos em quatro grupos 
experimentais:

- Grupo controle (WKY, $n=6)$ : os animais receberam diariamente apenas o veículo (óleo de girassol) utilizado para administração do óleo essencial, por via intraperitoneal, durante 30 dias;

- Grupo hipertenso (SHR, $n=6)$ : os animais receberam diariamente apenas o veículo (óleo de girassol) utilizado para administração do óleo essencial, por via intraperitoneal, durante 30 dias;

- Grupo normotenso tratado com óleo essencial (WKYP, $n=6$ ): os animais (WKY) receberam diariamente o óleo essencial $\left(10 \mathrm{mg} \mathrm{Kg}^{-1}\right)$ por via intraperitoneal, durante 30 dias de tratamento;

- Grupo hipertenso tratado com óleo essencial (SHRP, $n=6$ ): os animais ( $S H R$ ) receberam diariamente 0 óleo essencial $\left(10 \mathrm{mg} \mathrm{Kg}^{-1}\right)$ por via intraperitoneal, durante 30 dias de tratamento;

\section{Procedimentos cirúrgicos e registros hemodinâmicos}

Utilizou-se cateter de polietileno (PE 50 - Clay Adans $^{\circledR}$, USA), o qual se destinou à artéria femoral. Sob anestesia pelo pentobarbital sódico (50 $\mathrm{g} \mathrm{Kg}^{-1}$, via intra-peritoneal, Hypnol${ }^{\circledR}$, Cristália, Brasil), uma incisão na região inguinal, com posterior isolamento do plexo vásculo-nervoso, possibilitou a cateterização da aorta via artéria femoral. A terminação livre do cateter foi mantida preenchida com solução salina $(0,9 \%)$ e ocluída com pinos de aço inoxidável.

O cateter arterial femoral foi conectado a transdutores de pressão (Spectramed - Statham ${ }^{\circledR}$, P23XL, USA) através de cateter flexível, e os valores de registro da pressão arterial média (PAM), pulsátil (PAP) e Freqüência Cardíaca (FC) foram obtidos através de sistema computadorizado (Pentium MMX $233 \mathrm{MHz}$ ) e programa para aquisição de dados biológicos (BIOPAC ${ }^{\circledR}$ - BIOPAC Systems, Inc., Santa Barbara, Califórnia, USA, mod. MP 100A/série 94111065).

\section{Análise de hipertrofia do coração}

Ao final do protocolo experimental os animais foram sacrificados por injeção in bolus de anestésico. O coração foi isolado, lavado com solução fisiológica e o excesso de líquido foi removido com papel de filtro e em seguida pesado. $O$ ventrículo direito (VD) foi separado do ventrículo esquerdo (VE) e o septo interventricular foi considerado parte do VE (Andrade et al., 2007). A razão do peso ventricular pelo peso corporal (VE/PC ou VD/PC) foi usada como índice para a estimativa da hipertrofia cardíaca (Andrade et al., 2007).

\section{Protocolo experimental}

Os animais foram pesados e colocados em gaiolas individuais com livre acesso à água e ração. A dose de óleo essencial ministrada foi de $10 \mathrm{mg} \mathrm{Kg}^{-1}$
(Lahlou et al., 2003) e o veículo utilizado foi óleo de girassol (Dersani ${ }^{\circledR}$; Advanced products, Brasil).

Todos os animais foram pesados semanalmente. Ao final do tratamento, os animais foram anestesiados com pentobarbital sódico, para possibilitar o cateterismo da artéria femoral e os registros hemodinâmicos de Pressão Arterial Média (PAM) e Freqüência Cardíaca (FC). Ao final do experimento, os ratos foram sacrificados por meio de injeção in bolus de doses elevadas de anestésico. Em seguida, o coração foi retirado para obtenção do peso úmido e estimativa da hipertrofia cardíaca.

\section{Análise estatística}

Os valores das avaliações biológicas realizadas foram expressos como média \pm erro padrão da média (EPM). Para análise estatística dos valores basais de PAM e FC, peso corporal, peso das câmaras cardíacas, dos valores de VD/PC e VE/PC; os mesmos foram submetidos à análise de variância (ANOVA, uma via). A significância da diferença entre as médias foi determinada por teste post hoc pelo método de Fisher, ajustado para múltiplas comparações, com significância aceita acima de $5 \%$ $(p<0,05)$. Para análise estatística e apresentação gráfica dos resultados foram empregados os softwares GB-Stat (S.N.96003126) e Slide Write Plus (S.N.WSWP - C018529), respectivamente.

\section{RESULTADO}

\section{Análise da anatomia vegetal}

Empregando-se corte histológico no plano paradérmico da lâmina foliar, foram observadas epidermes adaxial e abaxial glabras com células poligonais de paredes retas, dispostas em fileiras, e estômatos paracíticos e tetracíticos em ambas as faces, concordando com Albuquerque \& Neves (2004). Já os cortes transversais do limbo foliar mostraram folha dorsiventral, revestida por epidermes unisseriadas, protegidas por cutícula mais espessa na face adaxial da lâmina foliar. Abaixo das epidermes ocorrem de um a três estratos de células hipodérmicas mucilaginosas e portadoras de gotas lipídicas, conforme previamente descrito por Albuquerque \& Neves (2004).

O clorênquima está organizado por uma camada de células de parênquima paliçádico e por quatro a cinco camadas de parênquima lacunoso. Entre as células do clorênquima ocorrem células mucilaginosas, muitas células isodiamétricas, oleíferas e idioblastos cristalíferos portadores de cristais prismáticos de oxalato de cálcio (Albuquerque \& Neves, 2004).

O sistema vascular é formado por feixes colaterais revestidos pela bainha de feixe de natureza 
parenquimática. As células do floema e da bainha de feixe apresentam gotas lipídicas e teste positivo para substâncias tânicas (Albuquerque \& Neves, 2004). Partindo da bainha até a epiderme, observam-se células fibrosas que constituem a extensão esclerenquimática da bainha.

\section{Característica física do óleo essencial e rendimento da extração \\ O óleo essencial de folhas secas de $A$.} zerumbetapresentou coloração amarelada e forte odor amadeirado refrescante. $O$ rendimento da extração do óleo essencial de folhas dessa espécie foi de 0,25 $\pm 0,03 \%(\mathrm{~m} / \mathrm{m})$. O coeficiente de variação $(\mathrm{CV}) \mathrm{de}$ extração foi de $12,40 \%$, indicando ter ocorrido pequena diferença entre as repetições de obtenção do óleo essencial. O CV representa o desvio padrão expresso como porcentagem da média, sendo comumente utilizada para estimar a precisão dos experimentos (Brasil, 2003).

\section{Análise Cromatográfica}

Pela análise da constituição química do óleo essencial de folhas de $A$. zerumbet, realizada por cromatografia gasosa acoplada à espectrometria de massas (CG-EM), foi possível identificar vários constituintes, sendo 14 as principais substâncias, todos de natureza terpênica, sete monoterpenos e sete sesquiterpenos (Tabela 1). O constituinte majoritário foi o monoterpeno terpinen-4-ol (37,45\%), seguido pelo sesquiterpeno óxido de cariofileno $(7,56 \%)$ e pelos monoterpenos trans-hidrato de sabineno $(6,61 \%)$ e 1,8-cineol (4,02\%) (Figura 1).

\section{Análise do peso corporal, do coração e da hipertrofia cardíaca}

Ao final do período de tratamento os animais dos grupos SHR e SHRP apresentaram relação VD/ PC $\left(0,55 \pm 0,02 \mathrm{mg} \mathrm{g}^{-1} ; 0,54 \pm 0,04 \mathrm{mg} \mathrm{g}^{-1}\right.$; respectivamente; $p<0,01)$ e VE/PC $(2,61 \pm 0,01 \mathrm{mg}$ $\mathrm{g}^{-1} ; 2,504 \pm 0,03 \mathrm{mg} \mathrm{g}^{-1}$; respectivamente; $\left.p<0,01\right)$ estatisticamente superiores quando comparados aos grupos controle com (WKYP: VD/PC $=0,54 \pm 0,03 \mathrm{mg}$ $\left.\mathrm{g}^{-1} ; \mathrm{VE} / \mathrm{PC}=2,17 \pm 0,04 \mathrm{mg} \mathrm{g}^{-1}\right)$ ou sem tratamento (WKY: VD/PC=0,54 $\pm 0,04 \mathrm{mg} \mathrm{g}^{-1} ; \mathrm{VE} / \mathrm{PC}=2,15 \pm$ $0,04 \mathrm{mg} \mathrm{g}^{-1}$ ). Além disso, o tratamento com OEAZ foi capaz de reduzir a relação VE/PC dos animais SHRP em comparação aos SHR ( $p<0,01)$ (Figura 2). O mesmo não foi observado para a relação VD/PC. $O$ resultado da análise do peso dos ventrículos direito e esquerdo, segue o mesmo padrão encontrado para a hipertrofia destas câmaras (Tabela 2). Também não foi observada diferença estatística entre os grupos experimentais no peso corporal inicial $(\mathrm{PCl})$ e no peso corporal final (PCF).
TABELA 1. Percentual (\%) e índice de Kovats (IK) dos constituintes do óleo essencial de folhas de $A$. zerumbet.

\begin{tabular}{lcc}
\hline Constituinte & $\%$ & IK $^{\mathbf{a}}$ \\
\hline 1,8-Cineol & 4,02 & 1034 \\
cis-Hidrato de sabineno & 3,55 & 1072 \\
trans-Hidrato de sabineno & 6,61 & 1102 \\
Terpinen-4-ol & 37,45 & 1188 \\
$\alpha$-Terpineol & 3,37 & 1196 \\
trans-Piperitol & 0,97 & 1212 \\
cis-Ascaridol & 1,60 & 1244 \\
trans-Nerolidol & 1,82 & 1568 \\
Óxido de cariofileno & 7,56 & 1590 \\
Epóxido de humuleno II & 1,01 & 1614 \\
$\gamma$-Eudesmol & 2,27 & 1637 \\
$\beta$-Eudesmol & 1,96 & 1657 \\
$\alpha$-Eudesmol & 1,90 & 1660 \\
Khusinol & 1,57 & 1678 \\
Outros & 13,33 & \\
\hline Total & 100,00 & \\
\hline & & \\
\hline
\end{tabular}

aÍndice de retenção calculado pela co-injeção de uma série homóloga de n-alcanos usando uma coluna capilar apolar DB-5, $30 \mathrm{~m}$.

\section{Efeito antihipertensivo}

O tratamento crônico de 30 dias com injeções in bolus intraperitoneais de OEAZ induziu a redução de PAM nos ratos SHRP $(160 \pm 7 \mathrm{~mm} \mathrm{Hg}$; $p<0,01)$ em relação aos animais SHR $(180 \pm 5 \mathrm{~mm}$ $\mathrm{Hg}$ ); porém os níveis pressóricos dos animais SHRP mantiveram-se diferentes dos animais controle (WKY= $106 \pm 2 \mathrm{~mm} \mathrm{Hg}$ e WKYP= $109 \pm 4 \mathrm{~mm} \mathrm{Hg} ; \mathrm{p}<0,05)$. Da mesma forma, e como esperado, os níveis de PAM dos animais SHR foram estatisticamente superiores aos dos ratos controle $(p<0,01)$ (Figura 3). Não houve diferença significativa nos valores de $\mathrm{FC}$ basal entre os grupos experimentais $(\mathrm{WKY}=320$ $\pm 15 ; \mathrm{WKYP}=310 \pm 12 ; \mathrm{SHR}=326 \pm 10 ; \mathrm{SHRP}=334$ \pm 14 b.p.m.). 


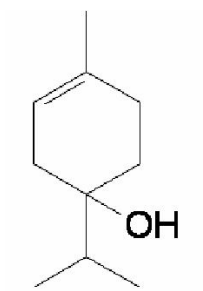

Terpinen-4-ol

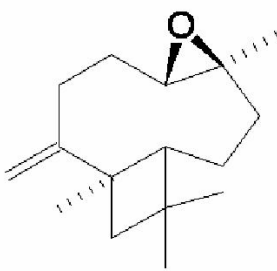

Óxido de cariofileno

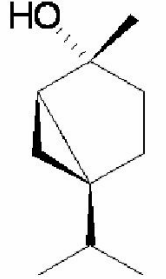

trans-Hidrato de sabineno

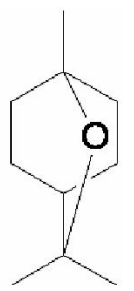

1,8-Cineol

FIGURA 1. Estrutura dos componentes majoritários encontrados no óleo essencial de folhas de $A$. zerumbet.

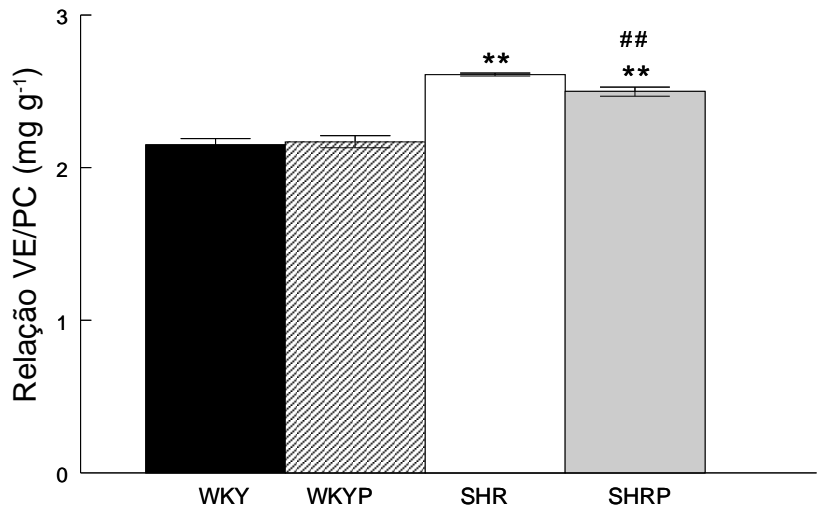

FIGURA 2. Efeito do tratamento com óleo essencial de $A$. zerumbet $\left(10 \mathrm{mg} \mathrm{kg}^{-1}\right)$ sobre o coração. Relação ventrículo esquerdo/peso corporal (VE/PC). Os valores estão expressos como a média \pm EPM. " $p<0,01$ em relação aos ratos controle (WKY e WKYP). ${ }^{\#} \mathrm{p}<0,01$ em relação aos animais SHR.

TABELA 2. Peso corporal dos animais experimentais antes e após o tratamento e dos ventrículos direito e esquerdo após o tratamento.

\begin{tabular}{ccccc}
\hline Grupo & PCI (g) & PCF (g) & VD (g) & VE (g) \\
\hline WKY & $202 \pm 7$ & $252 \pm 9$ & $0,13 \pm 0,04$ & $0,54 \pm 0,01$ \\
WKYP & $199 \pm 5$ & $255 \pm 7$ & $0,14 \pm 0,05$ & $0,57 \pm 0,02$ \\
SHR & $209 \pm 10$ & $243 \pm 10$ & $0,13 \pm 0,05$ & $0,63 \pm 0,01^{* *}$ \\
SHRP & $207 \pm 5$ & $249 \pm 11$ & $0,13 \pm 0,02$ & $0,60 \pm 0,03^{* \star \#}$
\end{tabular}

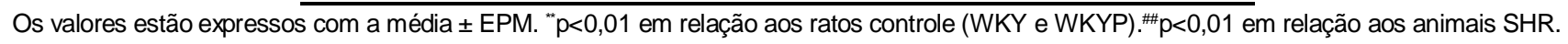

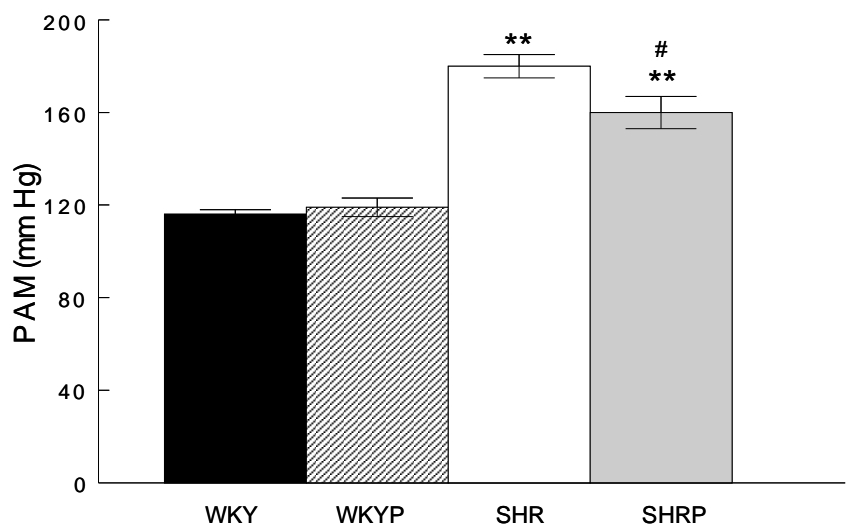

FIGURA 3. Valores basais da PAM nos grupos controle (WKY e WKYP) e nos animais hipertensos com (SHRP) ou sem tratamento (SHR). Dados expressos com a média \pm EPM. " $p<0,01$ em relação aos ratos controle (WKY e WKYP). ${ }^{\#} p<0,05$ em relação aos animais SHR. 


\section{DISCUSSÃO}

Todas as características observadas durante a análise da anatomia vegetal, do material empregado neste trabalho, estão em concordância com descrito previamente para a $A$. zerumbet (Zingiberaceae) (Albuquerque \& Neves, 2004).

$O$ perfil químico de OEAZ obtido nesse estudo difere do previamente descrito para as folhas de A. zerumbet (Zoghbi et al., 1999; Ali et al., 2002). Entretanto, está em concordância com a descrição do monoterpeno terpinen-4-ol como o componente majoritário (Zoghbi et al., 1999; Ali et al., 2002). Zoghbi et al. (1999) detectaram 1,8-cineol apenas nas flores da espécie, enquanto o limoneno, descrito como segundo componente majoritário por esses autores, não foi detectado no presente trabalho. Por outro lado, Ali et al. (2002) detectaram terpinen-4-ol, 1,8-cineol e b-pineno como os componentes majoritários do óleo essencial de folhas de $A$. zerumbet. A maturação foliar, a sazonalidade, o local e horário de coleta, o processo de secagem e o armazenamento, são fatores que influenciam na qualidade e composição de óleos essenciais (Oliveira et al., 2006; Stefanini et al., 2006), os quais poderiam explicar a diferença na composição química observada nesse trabalho com os dados previamente descritos.

$O$ efeito crônico antihipertensivo de OEAZ foi evidenciado nesse estudo, empregando-se modelo experimental dos ratos SHR. O efeito hipotensor agudo do óleo essencial de $A$. zerumbet foi descrito por Lahlou et al. (2003), e o efeito antihipertensivo crônico foi descrito para extrato hidroalcoólico de folhas dessa espécie (Moura et al., 2005), ambos utilizando o modelo de hipertensão DOCA-sal.

Lahlou et al. (2003) demonstraram que o terpinen-4-ol foi capaz de determinar, de maneira semelhante à observada no estudo com o OEAZ, redução da pressão arterial e efeito vasodilatador agudamente em animais DOCA-sal. No presente trabalho, o terpinen-4-ol apresentou-se como constituinte majoritário, podendo-se atribuir à presença deste componente o efeito anti-hipertensivo observado. Somado ao efeito hipotensor do constiuinte majoritário, outros componentes de OEAZ poderiam contribuir de forma sinérgica para a ação hipotensora de OEAZ. Por exemplo, foi descrita atividade hipotensora para o 1,8-cineol, atribuída ao efeito vasodilatador, em animais normotensos, no modelo de hipertensão DOCA-sal, dose de $10 \mathrm{mg}$ $\mathrm{Kg}^{-1}$, i.v. (Lahlou et al., 2002b). Empregando-se ratos Wistar anestesiados, 1,8-cineol apresentou efeito hipotensor na dose de 1-3 $\mathrm{mg} \mathrm{Kg}^{-1}$, i.v., sendo esse atribuído à capacidade de modular o sistema nervoso autônomo (Lahlou et al., 2002b).

Embora o OEAZ tenha exercido a ação antihipertensiva, não foi possível verificar normalização da pressão arterial quando comparadas às pressões arteriais médias dos animais normotensos. De acordo com estudos agudos conduzidos por Lahlou et al. (2003), foi possível observar redução da PAM, não sendo, todavia, relatada a normalização da mesma. Além disso, a administração crônica de $A$. zerumbet (extrato hidroalcoólico) em animais DOCA-sal ocasionou significativa redução das pressões sistólica, diastólica e média sem também observar a normalização (Moura et al., 2005).

Diversos estudos têm demonstrado que o OEAZ possui ação vasodilatadora (Lahlou et al., 2002a; Lahlou et al 2003; Moura et al., 2005). Em leitos mesentéricos, essa ação é independente de canais de potássio cálcio-dependente, voltagemdependente e ATP-dependente, mas que pode ocorrer via formação de óxido nítrico (NO) e formação de GMPcíclico, no músculo liso, como segundo mensageiro e, ainda, que deve possuir a participação de receptores de Bradicinina (via bradicinina/NO/GMPc) (Moura et al., 2005).

Dessa forma, a ativação dos receptores de bradicinina induz a produção de óxido nítrico pelo endotélio que, por sua vez, ativa a guanililciclase a produzir GMPc através de GTP (Garcia \& Incerpi, 2008). Como conseqüência do aumento intracelular deste segundo mensageiro, desencadeia-se uma sequência de fosforilações protéicas culminando na redução intracelular de cálcio e consequentemente no relaxamento da musculatura lisa vascular (Garcia \& Incerpi, 2008). Adicionalmente, Lahlou et al. (2002a, 2003) demonstraram que esta vasodilatação provavelmente não se deve a retirada simpática, corroborando a idéia de ação vasodilatadora direta para o OEAZ.

Outro resultado observado neste estudo foi a redução da hipertrofia cardíaca encontrada nos animais SHRP devido ao tratamento com OEAZ. Tem sido demonstrado que o tratamento antihipertensivo de ratos SHR determina a redução da hipertrofia cardíaca (Uggere et al., 2000; Andrade et al., 2008). Portanto, a redução do estresse de pressão sobre o coração devido à queda da pressão arterial nos ratos SHRP, poderia justificar, pelo menos em parte, a redução da hipertrofia cardíaca. Outra justificativa para a redução da hipertrofia cardíaca estaria relacionada à ativação da via bradicinina/NO/GMPc. Garcia \& Incerpi (2008), em estudo sobre atividade antihipertrófica do óxido nítrico, demonstraram que a redução intracelular de cálcio nas células musculares lisas endoteliais em função da ativação da via bradicinina/NO/GMPc, inativa enzimas mitogênicas e, dessa forma, melhora o perfil hipertrófico cardíaco.

Concluindo, o presente trabalho demonstra pela primeira vez, que o tratamento crônico com óleo essencial de $A$. zerumbet foi capaz de determinar redução da PAM e da hipertrofia cardíaca, no modelo de hipertensão experimental SHR, provavelmente pela 
presença dos componentes terpinen-4-ol e 1,8-cineol, o que poderia determinar melhoria da hemodinâmica cardiovascular dos animais SHRP, apesar da não normalização da pressão. Mais estudos são necessários para avaliar se doses maiores ou períodos de tratamento superiores aos realizados neste trabalho seriam suficientes para trazer os parâmetros hemodinâmicos para níveis de normalidade.

\section{AGRADECIMENTO}

Ao Centro Universitário Vila Velha - UVV e à Fundação Nacional de Desenvolvimento do Ensino Superior Particular (FUNADESP) pelo financiamento do trabalho. Ao prof. Dr. Luiz Cláudio de Almeida Barbosa, do Laboratório de Análise e Síntese de Agroquímicos (LASA - UFV), da Universidade Federal de Viçosa, pelas análises cromatográficas. Agradecemos à Pastoral da Saúde pela doação do material vegetal coletado.

\section{REFERÊNCIA}

ADAMS, R.P. Identification of essential oil components by gas chromatography mass spectroscopy. Carol Stream, Illinois: Allured. 1995. 469p.

ALBUQUERQUE, E.S.B.; NEVES, L.J. Anatomia foliar de Alpinia zerumbet (Pers.) Burtt \& Smith (Zingiberaceae). Acta Botanica Brasileira, v.18, n.1, p.109-21, 2004.

ALBUQUERQUE, U.P. et al. Medicinal and magic plants from a public market in northeastern Brazil. Journal of Ethnopharmacology, v.110, p.76-91, 2007.

ALI, S. et al. Comparison of the composition of essential oils of Alpinia and Hedychium species - essential oils of Fijian plants, part 1. Journal of Essential Oil Research, v.14, p.9-11, 2002.

ANDRADE, T.U. et al. Effect of enalapril treatment on the sensitivity of cardiopulmonary reflexes in rats with myocardial infarction. Clinical and Experimental Pharmacological and Physiology, v.34, n.7, p.606-11, 2007. ANDRADE, T.U. et al. Role of cardiac hypertrophy in reducing the sensitivity of cardiopulmonary reflex control of renal sympathetic nerve activity in spontaneously hypertensive rats. Clinical and Experimental Pharmacological and Physiology, v.35, n.9, p.1104-8, 2008.

BRANDÃO, M.G.L. Plantas medicinais. In: GUERRA, C.B.; BARBOSA, F.A.R. (Org.). Programa de Educação Ambiental na Bacia do Rio Piracicaba. Belo Horizonte: Editora da UFMG, 1996. p.173-93.

BRASIL. Ministério da Saúde. Agência Nacional de Vigilância Sanitária. Resolução RE no 899, de 29 de maio de 2003. Determina a publicação do "Guia para validação de métodos analíticos e bioanalíticos"; fica revogada a Resolução RE no 475, de 19 de março de 2002 Brasília, Diário Oficial da União, Poder Executivo, Brasília, DF, 2 jun. 2003.

BRASIL. Ministério da Saúde. Secretaria de Ciência, Tecnologia e Insumos Estratégicos. Programa nacional de plantas medicinais e fitoterápicos. Brasília: Ministério da Saúde, 2007. p.77
BUKATSH, F. Benerkemgemzeir doppelfarbeing astrablau-safranina. Microkosmos: Stuttgart, 1972. 255p.

GARCIA, J.A.D.; INCERPI, E.K. Fatores e mecanismos envolvidos na hipertrofia ventricular esquerda e o papel anti-hipertrófico do óxido nítrico. Arquivos Brasileiros de Cardiologia, v.90, n.6, p.443-50, 2008.

HOSTETTMANN, K.; MARSTON, A. Twenty years of research into medicinal plants: Results and perspectives Phytochemistry Review, v.1, p.275-85, 2002.

JOHANSEN, D.A. Plant microtechnique. New York: McGraw-Hill, 1940. 523p.

KRESS, W.J. et al. The molecular phylogeny of Alpinia (zingiberaceae): a complex and polyphyletic genus of gingers. American Journal of Botany, v.92, n.1, p.16778, 2005.

LAHLOU, S. et al. Antihypertensive effects of the essential oil of Alpinia zerumbet and its main constituent, terpinen4-ol, in DOCA-salt hypertensive conscious rats. Fundamental \& Clinical Pharmacology, v.17, n.3, p.32330, 2003.

LAHLOU, S. et al. Cardiovascular effects of the essential oil of Alpinia zerumbet leaves and its main constituent, terpinen-4-ol, in normotensive rats: role of the autonomic nervous system. Planta Medica, v.68, n.12, 1097-102, 2002a.

LAHLOU, S. et al. Cardiovascular effects of 1,8-cineole, a terpenoid oxide present in many plant essential oils, in normotensive rats. Canadian Journal of Physiology and Pharmacology, v.80, n.12, p.1125-31, 2002b.

LEAL-CARDOSO, J.H. et al. Effects of essential oil of Alpinia zerumbet on the compound action potential of the rat sciatic nerve. Phytomedicine, v.11, p.549-53, 2004. LEE, K.H. Current developments in the discovery and design of new drug candidates from plant natural product leads. Journal of Natural Products, v.67, p.273-83, 2004. MACIEL, M.A.M. et al. Plantas medicinais: A necessidade de estudos multidisciplinares. Química Nova, v.25, n.3, p.429-38, 2002.

MING, L.C. et al. Yield of essential oil of and citral content in different parts of lemongrass leaves (Cymbopogon citratus (DC.) Stapf.) Poaceae. Acta Horticulturae, v.1, n.426, p.555-9, 1996.

MOURA, R.S. et al. Antihypertensive and endotheliumdependent vasodilator effects of Alpinia zerumbet, a medicinal plant. Journal of Cardiovascular Pharmacology, v.46, n.3, p.288-94, 2005.

NATIONAL RESEARCH COUNCIL (U.S.A.). Committee on care and use of spontaneous hypertensive rats (SHR). Spontaneous hypertensive rats: guidelines for breeding, care and use. Institute of Laboratory Animal Resources (ILAR) News, v.19, n.3, p.G1-G20, 1976.

OKAMOTO, K.; AOKI K. Development of a strain of spontaneous hypertensive rats. Japanese Circulation Journal, v.27, p.282-93, 1963.

OLIVEIRA, C.J.; ARAÚJO, T.L. Plantas medicinais: usos e crenças de idosos portadores de hipertensão arterial. Revista Eletrônica de Enfermagem, v.9, n.1, p.93-105, 2007.

OLIVEIRA, E.C.P. et al. Identificação da época de coleta do óleo-resina de copaíba (Copaifera spp.) no município de Moju, PA. Revista Brasilera de Plantas Medicinais, v.8, n.3, p.14-23, 2006. 
PINHO, F.S.V.A. et al. Antinociceptive effects of the essential oil of Alpinia zerumbet on mice. Phytomedicine, v.12, p.482-6, 2005.

PIO CORREAA, M. Dicionário das plantas úteis do Brasil e das exóticas cultivadas. Rio de Janeiro: Ministério da Agricultura e IBDF, 1984. v.6, 4200p.

SKRUBIS, B.G. The drying of laurel leaves. Perfumer \& Flavorist, v.7, n.5, p.37-40, 1982.

STEFANINI, M.B. et al. Seed productivity, yield and composition of the essential oil of fennel Foeniculum vulgare var. dulcis in the season of the year. Revista Brasilera de Plantas Medicinais, v.8, n.esp., p.86-90, 2006.
UGGERE, T.A. et al. The cardiopulmonary reflexes of spontaneously hypertensive rats are normalized after regression of left ventricular hypertrophy and hypertension. Brazilian Journal of Medical and Biological Research, v.33, n.5, p.589-94, 2000.

WHO. World Health Organization. Traditional Medicine Strategy 2002 - 2005. Geneva: World Health Organization, 2002. 74p.

ZOGHBI, M. et al. Volatile constituents from leaves and flowers of Alpinia speciosa K. Schum., and A. purpurata (Viell.) Schum. Flavour and Fragrance Journal, v.14, p.411-4, 1999. 